\title{
Endogenous Levels of Polyamines under Water-Deficit Stress during Cotton's Reproductive Development
}

\author{
Dimitra A. Loka, Derrick M. Oosterhuis, Cristiane Pilon \\ Altheimer Laboratory, Department of Crop, Soil and Environmental Sciences, University of Arkansas, \\ Fayetteville, AR, USA \\ Email: dloka@uark.edu
}

Received 17 January 2015; accepted 8 February 2015; published 12 February 2015

Copyright (C) 2015 by authors and Scientific Research Publishing Inc.

This work is licensed under the Creative Commons Attribution International License (CC BY).

http://creativecommons.org/licenses/by/4.0/

(c) (i) Open Access

\begin{abstract}
Polyamines, putrescine (PUT), spermidine (SPD) and spermine (SPM) are implicated in plants' responses under conditions of abiotic stress. Previous research in other crops has indicated that polyamines and changes in their concentrations are associated with drought tolerance under conditions of water-deficit stress; however, no information exists on cotton (Gossypium hirsutum L.). Growth chamber experiments were conducted with two cotton cultivars differing in drought tolerance, ST5288B2F (drought-sensitive) and Siokra L23 (drought-tolerant) in order to investigate the distribution of free polyamines, the effect of water-deficit stress on the polyamine metabolism of cotton reproductive units and their subtending leaves as well as the possible relationship between polyamines and drought tolerance in cotton. Our results showed that cotton ovaries contained significantly higher levels of total free polyamines compared to their subtending leaves under both control and water stress conditions. Water-deficit stress significantly increased PUT concentrations in ST5288B2F, while SPM levels significantly decreased in Siokra L23. The results indicated that water-deficit stress significantly affected cotton polyamine metabolism in reproductive structures and their subtending leaves; however, no clear relationship between droughttolerance and changes in polyamine accumulation was established. Further research is needed to elucidate the mechanism according to which water-deficit stress affects polyamine metabolism.
\end{abstract}

\section{Keywords}

Cotton, Gas Exchange, Polyamine Levels, Reproductive Units, Water-Deficit Stress

\section{Introduction}

Polyamines (PA) are organic compounds, highly charged and found in bacteria, plants and animals. The most

How to cite this paper: Loka, D.A., Oosterhuis, D.M. and Pilon, C. (2015) Endogenous Levels of Polyamines under WaterDeficit Stress during Cotton's Reproductive Development. American Journal of Plant Sciences, 6, 344-354. 
frequently found PAs in plants are the diamine putrescine (PUT) and its derivatives, triamine spermidine (SPD) and tetramine spermine (SPM). PAs occur in free forms, conjugated with phenolic acids forms or bound to other low molecular weight compounds or macromolecules forms, and are implicated in a variety of plant functions, that include photosynthesis and stomatal conductance, enzyme activation and maintenance, cell division and differentiation, morphogenesis and embryogenesis as well as organogenesis ([1] [2] and references therein). Correct conformation of nucleic acids, membrane stabilization and ion channel regulation, gene expression and translation, hormone action mediation, as well as heat shock proteins and macromolecule synthesis are also characterized by PA participation [3]. However, the role of PAs in reproductive development appears to be more than mere participation due to the significant increase in their concentrations as plants transition from their vegetative to reproductive stage of growth ([2] and references therein). Experiments with PA-deficient mutants or mutants with unbalanced PA metabolism resulted in abnormal growth and flowering patterns as well as delayed flowering [3]. Flower induction also includes PA participation [1] along with flower initiation, pollination, fruit growth and ripening while sexual differentiation of tissues is reported to be strongly dependent on PA biosynthesis and catabolism. In addition, post-fertilization development has been reported to be regulated by PAs and numerous reports on PA and their role in stimulating fruit set and fruit development exist ([2] and references therein).

Investigations in a number of plant species has shown that changes in PA concentrations are a common plant response to a variety of abiotic stresses, including salinity, potassium deficiency, high or low temperatures and drought as well as biotic stresses ([2] and references therein). Experiments with cultivars differing in stress tolerance have shown that stress-tolerant plants have generally the ability to increase PA biosynthesis as a response to stress and enhance endogenous PA levels, and especially PUT, compared to the unstressed plants [4]. However, the opposite or no changes in PA levels have also been observed [5] [6]. Interestingly, apart from the endogenous PA, application of exogenous PAs has been reported to increase stress tolerance in plants [6], while application of PA biosynthesis inhibitors has the opposite effect [7]. Furthermore, research with transgenic plants transformed to increase PA production has reported that transformed plants have enhanced stress tolerance compared to their respective wild types [3].

Despite the extensive research on other crops, limited information on PA metabolism exists for cotton (Gossypium hirsutum L.) with the earliest reports being on the occurrences of uncommon polyamines (norspermidine, norspermine, pentamine, and hexamine) by Kuehn et al. [8] and Davidonis [9] on the distribution of polyamines (free, conjugated and bound) in both seeds and fibers. Bibi et al. [10] observed that nodal position of the flowers affected the distribution of free polyamines, with increasing nodal position resulting in decreasing polyamine concentrations, while heat stress resulted in significant decreases in both SPD and SPM levels of cotton ovaries, while free PUT levels remained unaffected [6]. However, application of exogenous PUT on heat-stressed cotton flowers resulted in significant increases in ovary PUT concentrations and seed set indicating that PUT could have an ameliorating effect on cotton under conditions of heat stress. Considering that drought is the major abiotic factor already affecting $30 \%$ of cultivated areas around the world and projections anticipate that water stress episodes are going to intensify in the future due to increased greenhouse gas concentrations, tools to help with drought-tolerant selection genotypes are greatly needed. Cotton is a relatively drought tolerant crop since its wild, perennial ancestors originated in hot and dry areas. However, cotton modern cultivars that are cultivated as annuals appear to be less drought-tolerant since water-deficit stress occurring at any stage of development, but especially during flowering, results in significant compromises in the morphology, physiology, metabolism and ultimately yield ([11] and references therein).

In order to investigate the effect of water-deficit stress on cotton polyamine metabolism, two cultivars differing in drought tolerance were used. Siokra L23, an okra leaf-type cultivar that has been shown to be drought tolerant [12] and high yielding under dry land conditions [13], while ST5288B2F a normal leaf-type cultivar grown widely in the Mississippi Delta area was considered to be the drought-sensitive cultivar based on variety trials results [14]. The objectives of this study were to monitor the changes in free PA levels in flower ovaries and their subtending leaves as well as determine whether free PAs are involved in drought tolerance.

\section{Materials and Methods}

Cotton (Gossypium hirsutum L.) cultivars ST5288B2F and Siokra L23 were planted in February 2011 at the Altheimer Laboratory, University of Arkansas into sixty 1 L pots containing Sunshine potting media mix\#1 (Sun- 
Gro Distribution Inc., Bellevue, WA). The growth chamber (Conviron PGW36, Conviron Inc., Winnipeg, Canada) equipped with incandescent and fluorescent lamps, was set for a 14-h photoperiod with a photosynthetic photon flux density (PPFD) of $800-850 \mu \mathrm{mol} \cdot \mathrm{m}^{-2} \cdot \mathrm{s}^{-1}$ and a relative humidity of $60 \%$. Cotton was grown under normal day/night temperatures of $32^{\circ} \mathrm{C} / 24^{\circ} \mathrm{C}$ and all pots received half-strength Hoagland's nutrient solution daily to maintain adequate nutrients and water. At flowering (8 weeks after planting, flowers at the $7^{\text {th }}$ node of the plants) container capacity was determined by the classical method of weighing the pots before, watering to saturation and allowing for excess drainage. Subsequently, pots were separated in two groups; Control pots received optimum quantity of water (equal to $100 \%$ of container capacity) all through the duration of the experiment while drought stress plants were allowed to dry slowly until plants reached wilting point (previously determined) for the first time. After that, water-stressed plants received $50 \%$ of optimum quantity of water (equal to $50 \%$ of container capacity) in order to keep them at a constant water-deficit stress for ten days. Stomatal conductance was also measured the next day in order to ensure the stress was kept at the same levels.

\subsection{Stomatal Conductance Measurements}

Stomatal conductance measurements were taken daily on the abaxial side from the fourth uppermost main-stem leaf $(n=10)$ and the leaf subtending to the flower, from 11:00 a.m. until 1:00 p.m. using a Decagon SC-1 Porometer (Decagon Inc., Pullman, WA). Three measurements on various areas of the leaf were taken then averaged and the results were expressed as $\mathrm{mmol} \cdot \mathrm{m}^{-2} \cdot \mathrm{s}^{-1}$.

\subsection{Photosynthesis Measurements}

A Li-Cor Model 6200 portable photosynthesis system (LICOR Inc., Lincoln, NE) was used to determine photosynthetic rates of the attached, fourth main-stem leaf from the terminal of the plant $(n=10)$. Measurements of photosynthesis were taken at 12:00 pm every two days after initiation of stress and the results were expressed as $\mu \mathrm{mol} \cdot \mathrm{m}^{-2} \cdot \mathrm{s}^{-1}$.

\subsection{Tissue Sampling and Free Polyamine Analysis}

First fruiting position cotton flowers from the $9^{\text {th }}$ node of each plant $(n=10)$ and their subtending leaves $(n=10)$ were sampled at 12:00 pm through the duration of the experiment. Cotton ovary and leaf tissues, 0.1 and $0.2 \mathrm{~g}$ respectively, were used for quantification of free polyamine concentrations and the extraction and analysis was performed according to Bibi et al. [6].

\subsection{Statistical Analysis}

A two factor factorial statistical analysis with the main factors being water regime and cultivar and fifteen replications, completely randomized, in each treatment was used to evaluate the results using JMP8 software (SAS Institute, Cary, NC). The experiment was repeated twice and similar trends were observed between the two studies so the results were pooled and the means were taken. Interactions and main effects were tested with Analysis of Variance (ANOVA) at $\alpha \leq 0.05$. When significant effects were detected, means were separated with Student's t-test $(\alpha \leq 0.05)$. For stomatal conductance results, the days of the experiment were not considered a factor and a single ANOVA was done for each day to compare differences among treatment combinations.

\section{Results}

\subsection{Leaf Stomatal Conductance}

Stomatal conductance of the fourth main-stem leaf from the terminal was significantly decreased under conditions of water-deficit stress for both cultivars (Figure 1). Day 0 of the experiment is the first day after plants had reached wilting point and had been watered with $50 \%$ of optimum quantity. Stomatal conductance was significantly different $(\mathrm{P} \leq 0.001)$ for days 0 - 10 of the experiment. However significant differences were observed between the two cultivars in the well-watered group, with Siokra L23 having consistently significantly ( $\leq$ 0.001) lower stomatal conductance than ST5288B2F for all the days of the experiment. A similar pattern was observed in the stomatal conductance of the subtending leaf (data not shown) with control stomatal conductance 


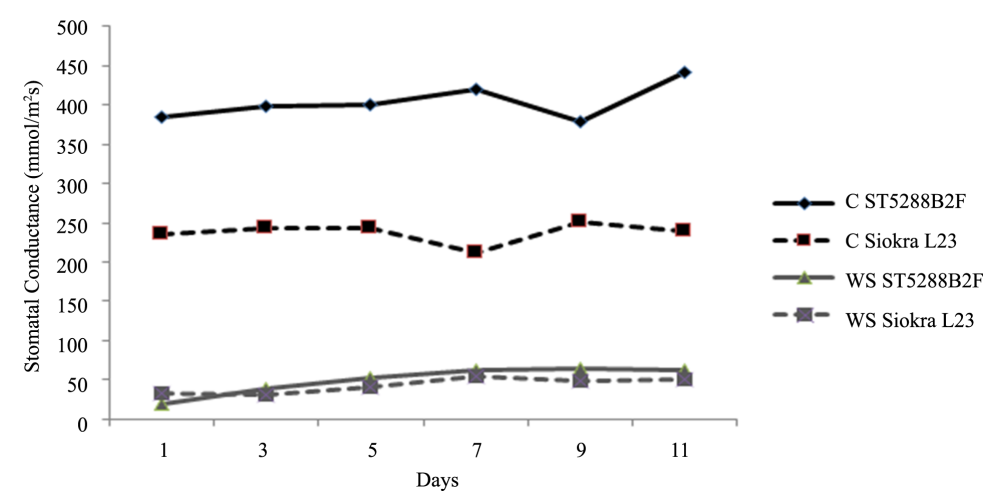

Figure 1. Stomatal conductance of cultivars ST5288B2F and Siokra L23 under well-watered (C) and water-stressed (WS) conditions $(n=10)$. Day 1 represents the first day after plants had reached wilting point and had been rewatered with $50 \%$ of optimum quantity and day 11 is the last day of the experiment. Points with the same letter were not significantly different $(\mathrm{P}=$ $0.05)$.

of both cultivars being significantly higher compared to the water-stressed plants. Subtending leaves of control plants of Siokra L23 additionally had significantly lower stomatal conductance compared to control plants of ST5288B2F (data not shown).

\subsection{Photosynthesis}

Photosynthetic rates were determined from a total of 10 replications from each cultivar in each treatment group and the results were analyzed separately for each date using a two factor factorial design with the main factors being water regime and cultivar. Results from both dates were similar so the results were pooled. No significant interaction $(\mathrm{P}=0.3039)$ was observed between the two main factors. Significant decreases in leaf photosynthetic rates were observed in the water-stressed plants of both cultivars compared to the control (Figure 2). Waterstressed plants of ST5288B2F had 45\% lower photosynthetic rates compared to their control and a similar pattern, but a higher decrease (59\%) was observed between control and water-stressed plants of Siokra L23.

\subsection{Polyamine Levels}

Significant differences were observed in the distribution of total polyamines with the ovaries containing higher concentrations of total polyamines compared to the leaves (Figure 3) under both control and water-deficit stress conditions.

The total polyamine levels in both leaf and ovaries of Siokra L23 remained unaffected under conditions of limited water supply compared to the control (Figure 3). Conversely, water-deficit stress resulted in a marked increase in the total polyamine content of the ovaries in the average drought-tolerant ST5288B2F; however, leaf total polyamine levels remained unaffected (Figure 3).

A significant increase in leaf PUT concentrations was observed for ST5288B2F where water-stressed plants containing 61 nmoles/gr FW PUT compared to 24 nmoles/gr FW of the control (Figure 4). Leaf PUT concentrations of Siokra L23 also increased; however, the increase was not statistically significant. Siokra L23 waterstressed plants contained comparably similar levels of PUT (63 nmoles/gr FW) to water-stressed ST5288B2F; however, control plants contained similar concentrations (55 nmoles/gr FW). A similar pattern was observed in ovary PUT concentrations (Figure 5). Water-stressed ovaries of ST5288B2F contained significantly higher concentrations of PUT compared to the control, while PUT in water-stressed Siokra L23was not significantly different compared to the control.

Water-deficit stress had no significant effect on the levels of leaf SPD for either cultivar (Figure 4). Both cultivars contained similar concentrations of SPD with ST5288B2F containing 146 nmoles/gr FW and 141 nmoles/ gr FW in control and water stress treatments, respectively, while Siokra L23 levels were 119 nmoles/gr FW and 125 nmoles/gr FW for control and water stress respectively. The SPD concentrations of the ovary (Figure 5), 


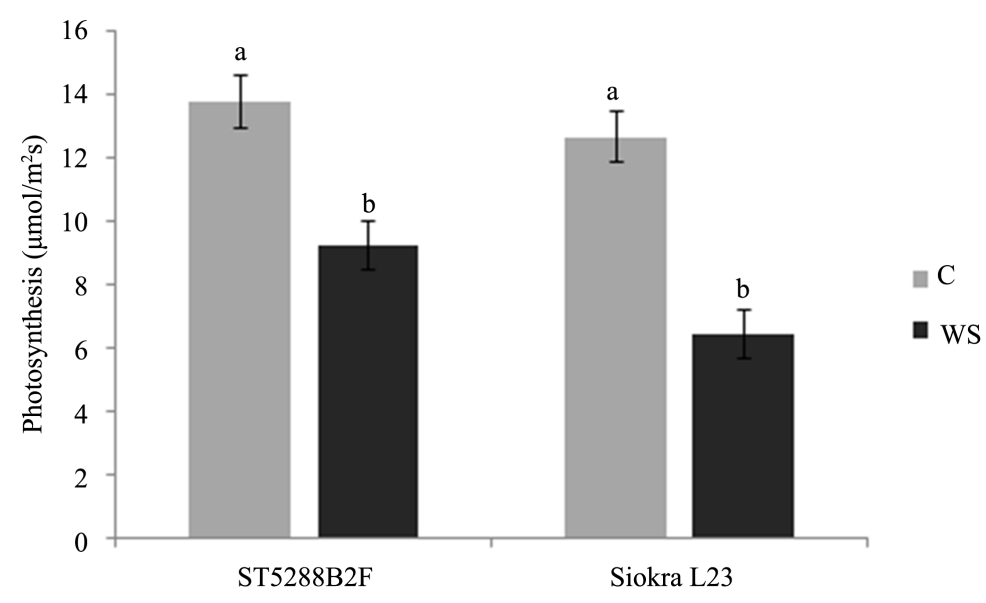

Figure 2. Leaf photosynthetic rates of ST5288B2F and Siokra L23 under well-watered (Control) and water-stressed (WS) conditions $(\mathrm{n}=10)$. Columns connected with the same letter are not significantly different $(P=0.05)$. Error bars represent \pm 1 standard error.

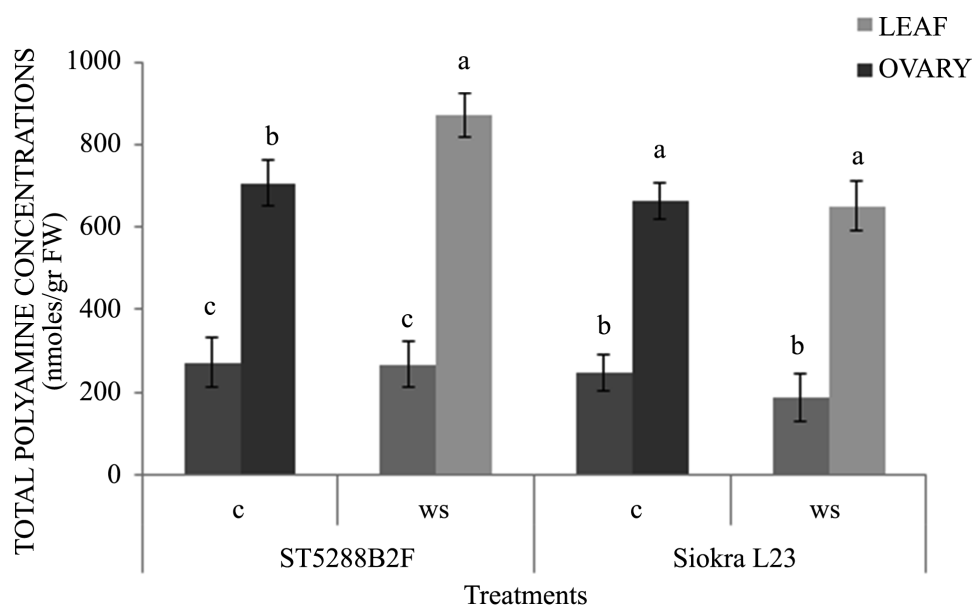

Figure 3. Distribution of total polyamine levels in the leaf and the ovary of cultivars ST5288B2F and Siokra L23 under well-watered (C) and waterstressed (WS) conditions $(n=10)$. Columns of the same cultivar connected with the same letter are not significantly different $(\mathrm{P}=0.05)$. Error bars represent \pm 1 standard error.

in Siokra L23 remained unaffected under conditions of water stress with control ovaries containing 336 nmoles/ gr FW SPD and water-stressed ovaries containing 334 nmoles/gr FW.

In contrast to SPD, water-deficit stress resulted in a significant decrease in leaf SPM levels of water-stressed Siokra L23 compared to the control, whereas no effect was observed in ST5288B2F (Figure 4). Concentrations of SPM in water-stressed Siokra L23 were nearly $40 \%$ lower compared to the control while leaf SPM levels of water-stressed ST5288B2F were 4\% higher compared to the control. Similarly to leaf SPM concentrations Siokra L23 ovary SPM concentrations were also decreased; however, not significantly compared to the control (Figure 5). ST5288B2F SPM levels of water-stressed ovaries were higher compared to the control but not statistically significant. However, water-stressed ST5288B2F ovaries contained significantly higher concentrations of SPM compared to water-stressed Siokra L23 ovaries.

\section{Discussion}

The results of our study showed that cotton free polyamine concentrations vary depending on the type of tissue. 


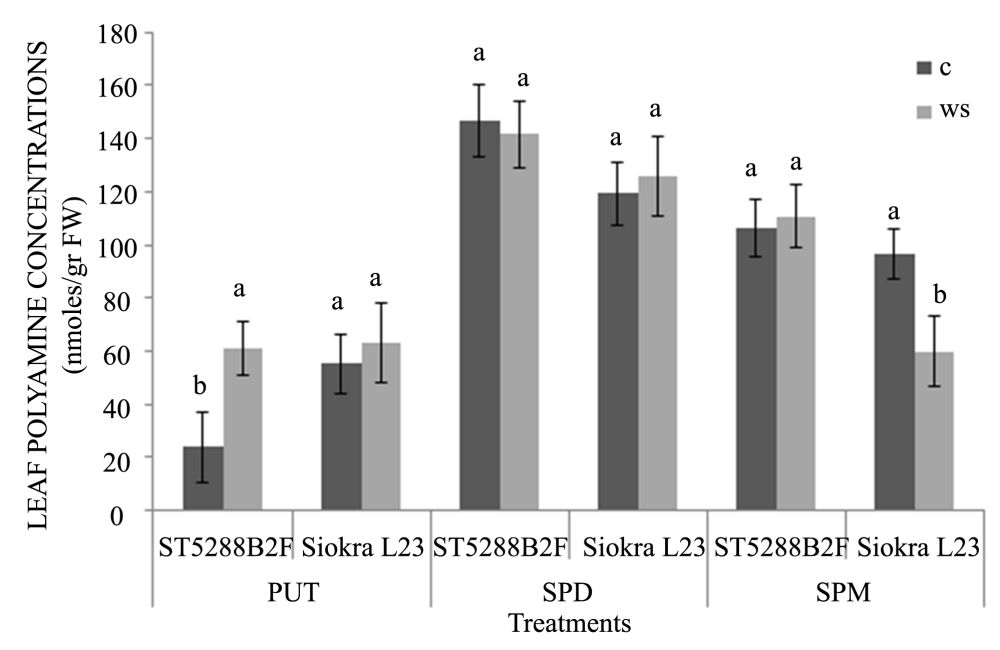

Figure 4. Distribution of leaf putrescine (PUT), spermidine (SPD) and spermine (SPM) concentrations of cultivars ST5288B2F and Siokra L23 under control (C) and water-deficit stress (WS) conditions $(n=10)$. Columns of the same polyamine connected with the same letter are not significantly different $(\mathrm{P}=0.05)$. Error bars represent \pm 1 standard error.

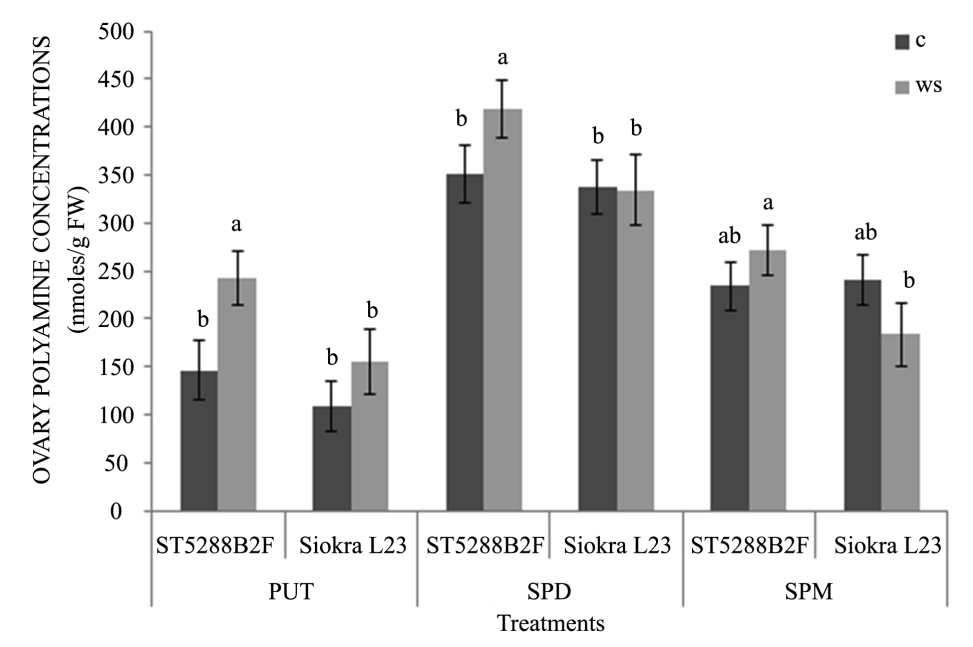

Figure 5. Distribution of ovary putrescine (PUT), spermidine (SPD) and spermine (SPM) concentrations of cultivars ST5288B2F and Siokra L23 under well-watered (C) and water-stressed (WS) conditions $(n=10)$. Columns of the same polyamine connected with the same letter are not significantly different $(\mathrm{P}=0.05)$. Error bars represent \pm 1 standard error.

Specifically, leaf polyamine concentrations were significantly lower compared to the ovaries. Cotton ovaries from both cultivars contained nearly twice the total polyamines concentrations compared to the leaves indicating that polyamines are closely associated with cotton's flowering mechanism. Significant increases in polyamine concentrations in reproductive structures as plants transition from vegetative to reproductive stages have been reported [1], and the importance of polyamine participation in flowering function has been established through experiments with polyamine deficient mutants that resulted in abnormal flowering structures or growth or even delayed flowering [3] [15]. Additionally, variations in polyamine concentrations have been reported depending on the species, the organ as well as the type of tissue analyzed [16] with the general trend being that increased polyamine concentrations occur inreproductive units compared to vegetative tissues [1]. However, in experiments with tomato (Lycopersicon esculentum) it was noticed that up-regulation of polyamine biosynthetic enzymes resulted in higher polyamine concentrations in both mature leaf tissues and ovaries [17]. Similar results 
were reported by Bae et al. [18] in cacao (Theobroma cacao) young leaf and ovary concentrations. Hence, we conclude that cotton's polyamine metabolism is more active in the reproductive organs compared to the vegetative tissues.

Focusing specifically on each cultivar, both leaf and ovary total polyamine levels of drought-tolerant Siokra L23 remained unaltered under conditions of limited water supply compared to the control. The drought-sensitive cultivar ST5288B2F, had significantly higher concentrations of total PAs in the water-stressed ovaries while leaf concentrations remained unaffected (Figure 3). Modulation of PA metabolism with concomitant changes in their concentrations have been observed in a variety of crops under conditions of water-deficit stress and PAs have been considered as protective agents due to their ability to function either as antioxidants or as signaling molecules for initiation of other protective mechanisms through their catabolism [16]. However, whether increases or decreases in their levels indicate enhanced drought-tolerance is still elusive. Lazcano-Ferrat and Lovatt [5], in field experiments with bean varieties (Phaseolus vulgaris and Phaseolus acutifolius) differing in drought tolerance observed that leaf total PA content of the drought-sensitive cultivar remained unaltered under conditions of water-deficit stress; however, leaf total PA concentrations of drought-tolerant cultivar were decreased compared to the control. Yang et al. [4] reported that both drought tolerant and sensitive rice (Oryza sativa) cultivars increased total PA concentrations; however, the drought tolerant ones were faster in their response. Similar results were reported by Yamaguchi et al. [19] in Arabidopsis, while Nayyar et al. [20] noticed that in drought-sensitive soybean (Glycine max) total PA content was decreased. Considering that PA response to abiotic stresses varies between species, tissues, duration and intensity of stress as well as developmental stages, we speculate that the stress, intensity or duration, imposed on the plants in our study (50\% of optimum quantity) was not sufficient to result in significant changes in total PA concentration of the drought-tolerant Siokra L23, while the opposite was observed in drought-sensitive ST5288B2F. Nevertheless, bearing in mind that each polyamine (PUT, SPD and SPM) appears to control different physiological functions as well as have diverse responses under conditions of stress depending on the species, the type of tissue and the developmental stage [16] we need to look at the effect of water-deficit stress in each polyamine individually.

Hence, in our study, PUT concentrations in both water-stressed leaf and ovary of ST52288B2F were significantly increased compared to the control while PUT levels in both tissues remained unaltered under conditions of water-deficit stress for the drought-tolerant Siokra L23 (Figure 4 \& Figure 5). Lefevre et al. [21] reported that diverse patterns in PUT accumulation under conditions of stress between photosynthetic and non-photosynthetic tissues; however, they investigated the effect of salt stress in rice shoots and roots. We assume that the incompatibility between the results is due to the difference of stress, species and tissues investigated.Increased leaf PUT concentrations have been associated with decreased drought tolerance in wheat (Triticum aestivum) [7] as well as decreased salt tolerance in rice [19] which is in partial agreement with the results of our study since higher levels than control were observed in both cultivars; however, significant increases were only for ST5288B2F. Conversely, Nayyar et al. [20] reported that PUT levels increased in drought tolerant chickpea (Cicer arietinum), while they decreased in more drought sensitive soybean. Yang et al. [4] reported that PUT levels in both drought tolerant and sensitive rice cultivars were increased; however, a lag in time was observed in the drought sensitive ones. In addition, Alcazar et al. [22] reported that transgenic Arabidopsis plants transformed to over-express PUT were more drought tolerant compared to wild type plants and this was attributed to the decrease in stomatal conductance, which was similar to our results. Water-stressed leaves of ST5288B2F had similar stomatal conductance with water-stressed leaves of Siokra L23 and a similar pattern was observed in photosynthetic rates. Interestingly, however, control leaves of Siokra L23 had significantly lower stomatal conductance compared to control ST5288B2F, while leaf PUT concentrations of control and water-stressed droughttolerant Siokra L23 were similar to those of ST5288B2F under water stress. Significantly lower stomatal conductance of okra isolines compared to normal under well-watered conditions has been reported by Pettigrew et al. [23] and the difference was attributed to the lower abaxial stomatal density observed between the okra and the normal isolines. Additionally, Pettigrew et al. [23] in field experiments as well as Nepomuceno et al. [12] in experiments with PEG-induced water stress reported higher photosynthetic rates for okra isolines compared to normal isolines at similarly low water potentials, which was not observed in our study since Siokra L23 had significantly lower photosynthetic rates compared to ST5288B2F under water-deficit stress conditions. We speculate that the differential responses in photosynthetic rates are due to the differences in the stress conditions between the various studies. Additionally, even though stomatal density was not measured in our experiments, we assume that in cotton PUT levels affect stomatal conductance under both normal and water-deficit conditions 
with high concentrations inducing stomatal closure, which is in accordance with Alcazar et al. [22]. The insignificant increase in PUT levels of drought-tolerant Siokra L23 could be attributed to the already high levels of PUT and the stress duration or intensity not being enough to trigger PUT biosynthesis [24].

Similarly to leaf PUT levels, ovary PUT levels of both ST5288B2F and Siokra L23 were increased under conditions of limited water supply, however only significantly for the ST5288B2F (Figure 5). Conversely, Bibi et al. [6] reported that PUT concentrations of heat-stressed ovaries were similar to those of the control. Nayyar et al. [20] observed increases in PUT levels of flowers and pods in both drought-tolerant and sensitive chickpea cultivars, however drought-sensitive cultivars had a faster response to the stress compared to the drought-tolerant ones. This differential response was attributed by the authors to the ability of drought-tolerant cultivars to preserve high water potentials under water-deficit stress. Even though water potentials were not monitored in our study, Siokra L23 has been reported to have significantly higher leaf water potential under conditions of stress compared to other cultivars [25]. Hence, we assume that the insignificant increase in ovary PUT levels of water-stressed Siokra L23 in our study is a result either of the low intensity of stress imposed to the plants, which was not enough to trigger PUT biosynthesis, according to the threshold model suggested by Cappell et al. [24] or/and Siokra L23 ability to maintain adequate water status under conditions of stress.

Triamine SPD derives directly from PUT by addition of one aminopropyl group. Associations between high SPD and enhanced drought-tolerance have been observed in a variety of crops [4] [19] [20], while exogenous application of SPD or over-expression of SPD biosynthesis genes in transformed plants have resulted in higher concentrations of SPD and enhancement of drought tolerance [26] suggesting that SPD plays a major role in protecting plants from water stress. However, leaf SPD concentrations in our study were observed to remain unaltered under conditions of water-deficit stress for both cultivars (Figure 4). Alcazar et al. [22] also reported no change in SPD concentrations of water-stressed wild-type or transformed to over-produce PUT Arabidopsis plants, no matter the increases in PUT levels. The authors suggested that SPD biosynthesis is under tighter control compared to PUT, whereas Capell et al. (24) attributed the lack of changes in SPD concentrations in rice drought-tolerant cultivars to the fact that the level of stress applied was insufficient to initiate PUT biosynthesis and further synthesis of SPD. Contrary to Alcazar et al. [22], who suggested that PUT controls stomatal control more effectively than SPD or SPM, Liu et al. [27] observed that SPD is more effective in inducing leaf stomatal closure on wheat. Nevertheless, this was not observed in our study. Leaf stomatal conductance rates were similar for both cultivars under conditions of water stress and significantly lower compared to the control while their SPD levels were similar under both control and water stress conditions. Zhang et al. [28] reported that exogenous application resulted in increased photosynthetic rates in both drought sensitive and tolerant cucumber cultivars. In our study, photosynthetic rates of water-stressed Siokra L23 were significantly lower not only compared to the control but also compared to water-stressed ST5288B2F (Figure 2). Hence, we assume that in cotton, SPD is not as effective as PUT in modulating stomatal function. We speculate that regarding average drought tolerant ST5288B2F, either the increases in PUT concentrations did not surpass a certain threshold in order to trigger SPD biosynthesis or that the synthesis rates of SPD were very slow resulting in no significant change as it has been shown previously by Flores and Galston [29], whereas the insignificant increase in PUT levels of drought tolerant Siokra L23 were definitely insufficient to result in changes in SPD levels.

Water-stressed ovary SPD concentrations remained similar to the control in Siokra L23, as well as the control in ST5288B2F, whereas water-stressed ST5288B2F ovaries contained significantly higher SPD levels (Figure 4 \& Figure 5). Increases in both flowers and early pods of chickpea in SPD concentrations of drought-tolerant as well as drought-sensitive cultivars were observed also by Nayyar et al. [20]. However, similarly to their PUT pattern drought-tolerant cultivars increased their SPD levels later into the stress, while drought-sensitive ones at the onset of the stress which leads us to assume that drought-tolerant Siokra L23 might need a more extended period of stress or a more intensive stress in order to increase its SPD concentrations, while the opposite is assumed for average tolerant ST5288B2F. In accordance with our assumption is the threshold model suggested by Capell et al. [24] where contrary to the drought-tolerant, drought-sensitive rice cultivars initiated SPD synthesis at the imposition of the stress.

Similarly to SPD, SPM has also been attributed significant roles regarding plant defense under conditions of abiotic stress. Water-deficit stress has been observed to result in increased levels of SPM in drought-tolerant cultivars or species [4] [19] [20]. In support of these observations, SPM-deficient mutants have been reported to be more susceptible to drought [19] while exogenous application of SPM has been observed to enhance drought tolerance [26] [30] due to its protective role in lipid peroxidation of membranes. However, in our study wa- 
ter-stressed leaf SPM levels of drought-tolerant Siokra L23 were significantly decreased compared to the control, while no significant change was observed in leaf SPM levels of ST5288B2F (Figure 4). Similar decreases in leaf SPM concentrations under water stress were also reported for the drought tolerant Phaseolus acutifolius while for drought-sensitive Phaseolus vulgaris SPM levels remained unaffected [5]. In their study, the observed decrease in SPM levels was attributed to the inability of $P$. acutifolius to use the accumulated nitrogen pools of the plant, while Capell et al. [31] in experiments with PEG-induced water stress on oat protoplasts suggested that SPM decreased due to the activation of the catabolic pathway. Alcazar et al. [22] speculated that the reduced SPM in Arabidopsis were due to the PUT concentrations not increasing over a threshold level and hence not initiating SPM synthesis, which is in accordance with our results. In addition, the low leaf SPM levels of Siokra L23 observed in our study, resulted in a significant decrease in its photosynthetic rates not only compared to the control but also compared to water-stressed ST5288B2F, indicating that SPM levels significantly modulate photosynthetic function in cotton. In support of this observation, Besford et al. [32] reported that exogenous application of SPM decreased chlorophyll degradation and protected Rubisco under conditions of water stress, while increased levels of SPM were associated with enhanced photosynthesis [33]. Contrary to Liu et al. [27] and Farooq et al. [26] SPM did not appear to have an important role in stomatal function since water-stressed ST5288B2F had similar stomatal conductance rates as water-stressed Siokra L23 even though it contained significantly higher levels of SPM.

Ovary SPM levels were similar to the leaf SPM content pattern. Under well watered conditions, SPM concentrations of the ovaries were similar for both cultivars however, water-stressed Siokra L23 contained significantly lower concentrations of SPM compared to ST5288B2F (Figure 5). Decreases in SPM content of cotton ovaries were also reported by Bibi et al. [6] under conditions of heat stress, whereas Nayyar et al. [20] contrastingly reported increases in SPM levels of both drought tolerant and sensitive cultivars of chickpea under conditions of water-deficit stress. In their experiment however, both SPD and PUT ovary concentrations were increased under stress. Since ovary levels of SPD and especially PUT of Siokra L23 remained fairly unaltered under conditions of water-deficit stress, while a significant increase was observed in PUT levels of water-stressed ST5288B2F, we speculate that the differential response in ovary SPM levels between the two cultivars is due to the PUT not reaching the levels necessary to activate SPD and SPM synthesis, a suggestion made also by Alcazar et al. [22].

\section{Conclusion}

In conclusion, the results of our study indicated that polyamines in cotton accumulated in higher concentrations in the reproductive structures compared to the vegetative tissues. Total polyamine concentrations were not shown to be affected significantly by water-deficit stress conditions; however the opposite was observed when each polyamine concentration was analyzed individually. Changes in leaf polyamine levels, PUT and SPM especially, suggested an association between leaf gas exchange functions and polyamine metabolism. The changes in the ovary polyamine levels, especially in PUT and SPD, indicated that polyamine metabolism of cotton reproductive units was far more responsive/sensitive to water-deficit stress and changes in ovary polyamine levels could be used as markers for drought tolerance, since increased PUT and SPD levels were noted in the drought sensitive cultivar. We conclude that polyamines play an important role in cotton protection under adverse environmental conditions and changes in their concentrations, especially in the reproductive units, can be used as potential markers for selection of drought tolerant cultivars. However, further research is needed in order for a clearer cause-effect relationship to be established.

\section{References}

[1] Kakkar, R.K. and Sawhney, V.P. (2002) Polyamine Research in Plants: A Changing Perspective. Physiologia Plantarum, 116, 281-292. http://dx.doi.org/10.1034/j.1399-3054.2002.1160302.x

[2] Oosterhuis, D.M. and Loka, D. (2012) Polyamines and Cotton Flowering. In: Oosterhuis, D.M. and Cothren, J.T., Eds., Flowering and Fruiting of Cotton, Publ. Cotton Foundation, Memphis, 109-132

[3] Alcazar, R., Altabella, T., Marco, F., Bortolotti, C., Reymond, M., Koncz, C., Carrasco, P. and Tiburcio, A.F. (2010) Polyamines: Molecules with Regulatory Functions in Plant Abiotic Stress Tolerance. Planta, 231, 1237-1249. http://dx.doi.org/10.1007/s00425-010-1130-0

[4] Yang, J., Zhang, J., Liu, K., Wang, Z. and Liu, L. (2007) Involvement of Polyamines in Drought Resistance of Rice. Journal of Experimental Botany, 58, 1545-1555. http://dx.doi.org/10.1093/jxb/erm032 
[5] Lazcano-Ferrat, I. and Lovatt, C.J. (1999) Relationship between Relative Water Content, Nitrogen Pools and Growth of Phaseolus vulgaris L. and P. acutifolius, A. Gray during Water Deficit. Crop Science, 39, 467-475. http://dx.doi.org/10.2135/cropsci1999.0011183X0039000200028x

[6] Bibi, A.C., Oosterhuis, D.M. and Gonias, E.D. (2010) Exogenous Application of Putrescine Ameliorates the Effect of High Temperature in Gossypium hirsutum L. Flowers and Fruit Development. Journal of Agronomy and Crop Science, 196, 205-211. http://dx.doi.org/10.1111/j.1439-037X.2009.00414.X

[7] Liu, H.P., Dong, B.H., Zhang, Y.Y., Liu, Z.P. and Liu, Y.L. (2004) Relationship between Osmotic Stress and the Levels of Free, Conjugated and Bound Polyamines in Leaves of Wheat Seedlings. Plant Science, 166, 1261-1267. http://dx.doi.org/10.1016/j.plantsci.2003.12.039

[8] Kuehn, G.D., Rodriguez-Garay, B., Bagga, S. and Phillips, G.C. (1990) Novel Occurrence of Uncommon Polyamines in Higher Plants. Plant Physiology, 94, 855-857. http://dx.doi.org/10.1104/pp.94.3.855

[9] Davidonis, G. (1995) Changes in Polyamine Distribution during Cotton Fiber and Seed Development. Journal of Plant Physiology, 145, 108-112. http://dx.doi.org/10.1016/S0176-1617(11)81855-9

[10] Bibi, A, C., Ooosterhuis, D.M., Gonias, E.D. and Mattice, J.D. (2012) Nodal Distribution of Free Polyamines in Cotton Ovaries Determined by HPLC. Journal of Agricultural Science, 150, 365-372. http://dx.doi.org/10.1017/S0021859611000633

[11] Loka, D. and Oosterhuis, D.M. (2012) Water Stress and Reproductive Development in Cotton. In: Oosterhuis, D.M. and Cothren, J.T., Eds., Flowering and Fruiting in Cotton, Publ. Cotton Foundation, Memphis, 51-57.

[12] Nepomuceno, A.L., Oosterhuis, D.M. and Stewart, J.M. (1998) Physiological Responses of Cotton Leaves and Roots to Water Deficit Induced by Polyethelene Glycol. Environmental and Experimental Botany, 40, 29-41. http://dx.doi.org/10.1016/S0098-8472(98)00018-5

[13] Stiller, W.N., Read, J.J., Constable, G.A. and Reid, P.A. (2005) Selection for Water Use Efficiency Traits in a Cotton Breeding Program: Cultivar Differences. Crop Science, 45, 1107-1113. http://dx.doi.org/10.2135/cropsci2004.0545

[14] Wheelus, A.D., Ritchie, G.L., Sexton, L. and Ford, J. (2009) Screening Water Stress in Multiple Cotton Varieties. Georgia Cotton Research and Extension Report, 78-82.

[15] Hanzawa, Y., Imai, A., Michael, A.J., Komeda, Y. and Takahashi, T. (2002) Characterization of the Spermidine Synthase-Related Gene Family in Arabidopsis thaliana. FEBS Letters, 527, 176-180. http://dx.doi.org/10.1016/S0014-5793(02)03217-9

[16] Minocha, R., Majumdar, R. and Minocha, S.C. (2014) Polyamines and Abiotic Stress in Plants: A Complex Relationship. Frontiers Plant Science, 5, 1-17. http://dx.doi.org/10.3389/fpls.2014.00175

[17] Alabadi, D., Aguero, M.S., Perez-Amador, M.A. and Carbonell, J. (1996) Arginase, Arginine Decarboxylase, Ornithine Decarboxylase, and Polyamines in Tomato Ovaries. Plant Physiology, 112, 1237-1244.

[18] Bae, H., Kim, S.H., Kim, M.S., Sicher, R.C., Lary, D., Strem, M.D., Natarajan, S. and Bailey, B.A. (2008) The Drought Response of Theobroma cacao and the Regulation of Genes Involved in Polyamine Biosynthesis by Drought and Other Stresses. Plant Physiology and Biochemistry, 46, 174-188. http://dx.doi.org/10.1016/j.plaphy.2007.10.014

[19] Yamaguchi, K., Takahashi, Y., Berberich, T., Imai, A., Takahashi, T., Michael, A.J. and Kusano, T. (2007) A Protective Role for the Polyamine Spermine against Drought Stress in Arabidopsis. Biochemical and Biophysical Research Communications, 352, 486-490. http://dx.doi.org/10.1016/j.bbrc.2006.11.041

[20] Nayyar, H., Kaur, S., Kumar, S., Singh, K.J. and Dhir, K.K. (2005) Involvement of Polyamines in the Contrasting Sensitivity of Chickpea (Cicer arietinum L.) and Soybean (Glycine max L. Merrill) to Water-Deficit Stress. Botanical Billboard Academia Sinica, 46, 333-338.

[21] Lefevre, I., Gratia, E. and Lutts, S. (2001) Discrimination between the Ionic and Osmotic Components of Salt Stress in Relation to Free Polyamine Level in Rice (Oryza sativa). Plant Science, 161, 943-952. http://dx.doi.org/10.1016/S0168-9452(01)00485-X

[22] Alcazar, R., Planas, J., Saxena, T., Zarza, X., Bortolotti, C., Cuevas, J., Bitrian, M., Tiburcio, A.F. and Altabella, T. (2010) Putrescine Accumulation Confers Drought Tolerance in Transgenic Arabidopsis Plants Over-Expressing the Homologous Arginine decarboxylase 2 Gene. Plant Physiology and Biochemistry, 48, 547-552. http://dx.doi.org/10.1016/j.plaphy.2010.02.002

[23] Pettigrew, W.T. (2004) Physiological Consequences of Moisture Deficit Stress in Cotton. Crop Science, 44, 1265-1272. http://dx.doi.org/10.2135/cropsci2004.1265

[24] Capell, T., Bassle, L. and Christou, P. (2004) Modulation of the Polyamine Biosynthetic Pathway in Trasngenic Rice Confers Tolerance to Drought Stress. Proceedings of the National Academy of Science of the USA, 101, 9909-9914. http://dx.doi.org/10.1073/pnas.0306974101

[25] Voloudakis, A.E., Kosmas, S.A., Tsakas, S., Eliopoulos, E., Loukas, M. and Kosmidou, K. (2002) Expression of Se- 
lected Drought-Related Genes and Physiological Response of Greek Cotton Varieties. Functional Plant Biology, 29, 1237-1245. http://dx.doi.org/10.1071/PP01253

[26] Farooq, M., Wahid, A. and Lee, D.J. (2008) Exogenous Applied Polyamines Increase Drought Tolerance of Rice by Improving Leaf Water Status, Photosynthesis, and Membrane Properties. Acta Physiologiae Plantarum, 31, 937-945.

[27] Liu, K., Fu, H., Bei, Q. and Luan, S. (2000) Inward Potassium Channel in Guard Cells as a Target of Polyamine Regulation of Stomatal Movements. Plant Physiology, 124, 1315-1325. http://dx.doi.org/10.1104/pp.124.3.1315

[28] Zhang, R.H., Li, J., Guo, S.R. and Tezuka, T. (2009) Effects of Exogenous Putrescine on Gas Exchange Characteristics and Chlorophyll Fluorescence of NaCl-Stressed Cucumber Seedlings. Photosynthesis Research, 100, 155-162. http://dx.doi.org/10.1007/s11120-009-9441-3

[29] Flores, H.E. and Galston, A.W. (1984) Osmotic-Stress Induced Accumulation in Cereal Leaves. I. Physiological Parameters of the Response. Plant Physiology, 75, 102-109. http://dx.doi.org/10.1104/pp.75.1.102

[30] Pang, X.M., Zhang, Z.Y., Wen, X.P., Ban, Y. and Moriguchi, T. (2007) Polyamines, All-Purpose Players in Response to Environmental Stresses in Plants. Plant Stress, 1, 173-188.

[31] Capell, T., Campos, J.L. and Tiburcio, A.F. (1993) Antisenescence Properties of Guazatine in Osmotically Stressed Oat Leaves. Phytochemistry, 32, 785-788. http://dx.doi.org/10.1016/0031-9422(93)85205-6

[32] Besford, R.T., Richardson, C.M., Campos, J.L. and Tiburcio, A.F. (1993) Effect of Polyamines on Stabilization of Molecular Complexes in Thylakoid Membranes of Osmotically Stressed Oat Leaves. Planta, 189, 201-206. http://dx.doi.org/10.1007/BF00195077

[33] Islam, M.A., Blake, T.J., Kocacinar, F. and Lada, R. (2003) Ambiol, Spermine and Aminoethoxyvinylglycine Prevent Water Stress and Protect Membrane in Pinus strobus under Drought. Trees Structure and Function, 17, 227-235. 
Scientific Research Publishing (SCIRP) is one of the largest Open Access journal publishers. It is currently publishing more than 200 open access, online, peer-reviewed journals covering a wide range of academic disciplines. SCIRP serves the worldwide academic communities and contributes to the progress and application of science with its publication.

Other selected journals from SCIRP are listed as below. Submit your manuscript to us via either submit@scirp.org or Online Submission Portal.
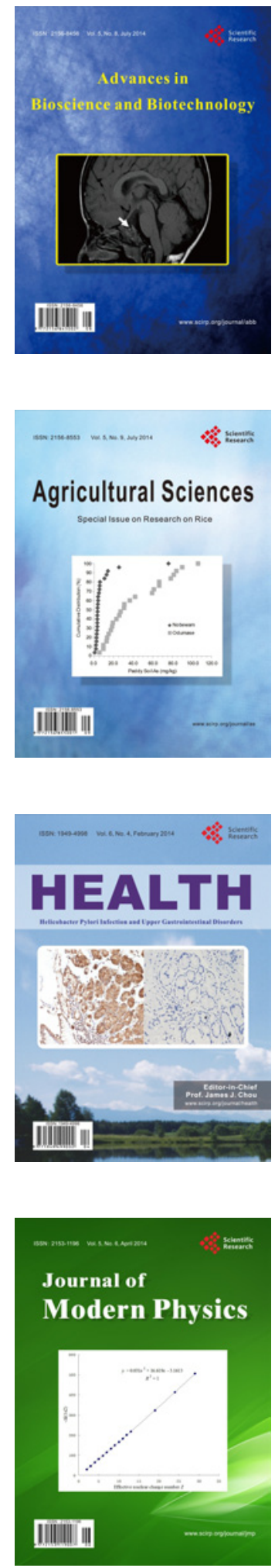
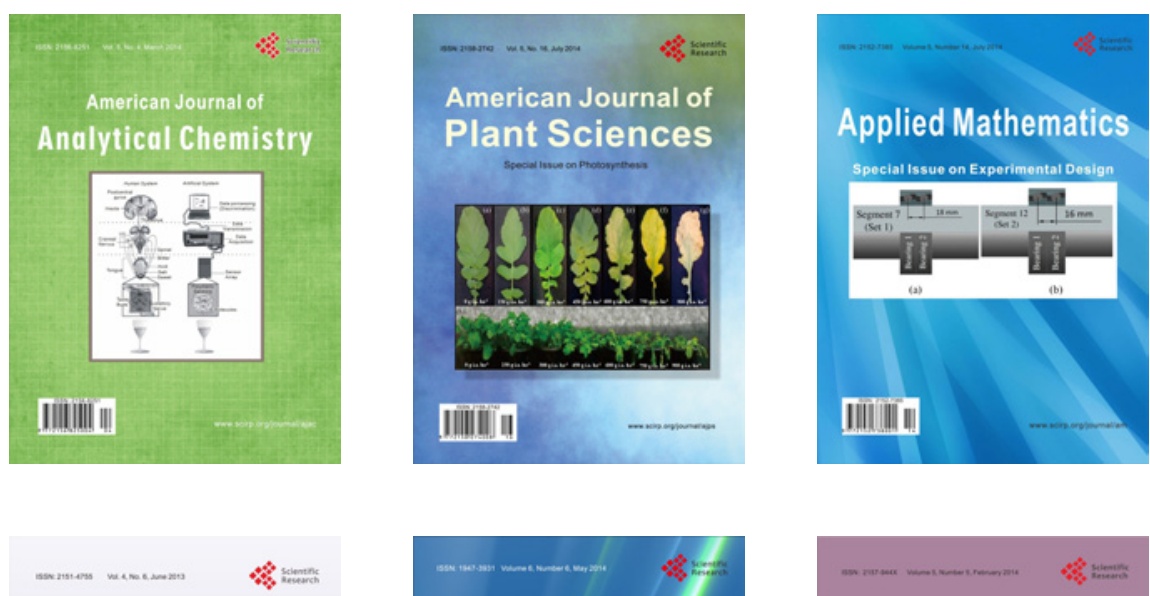

Creative Education
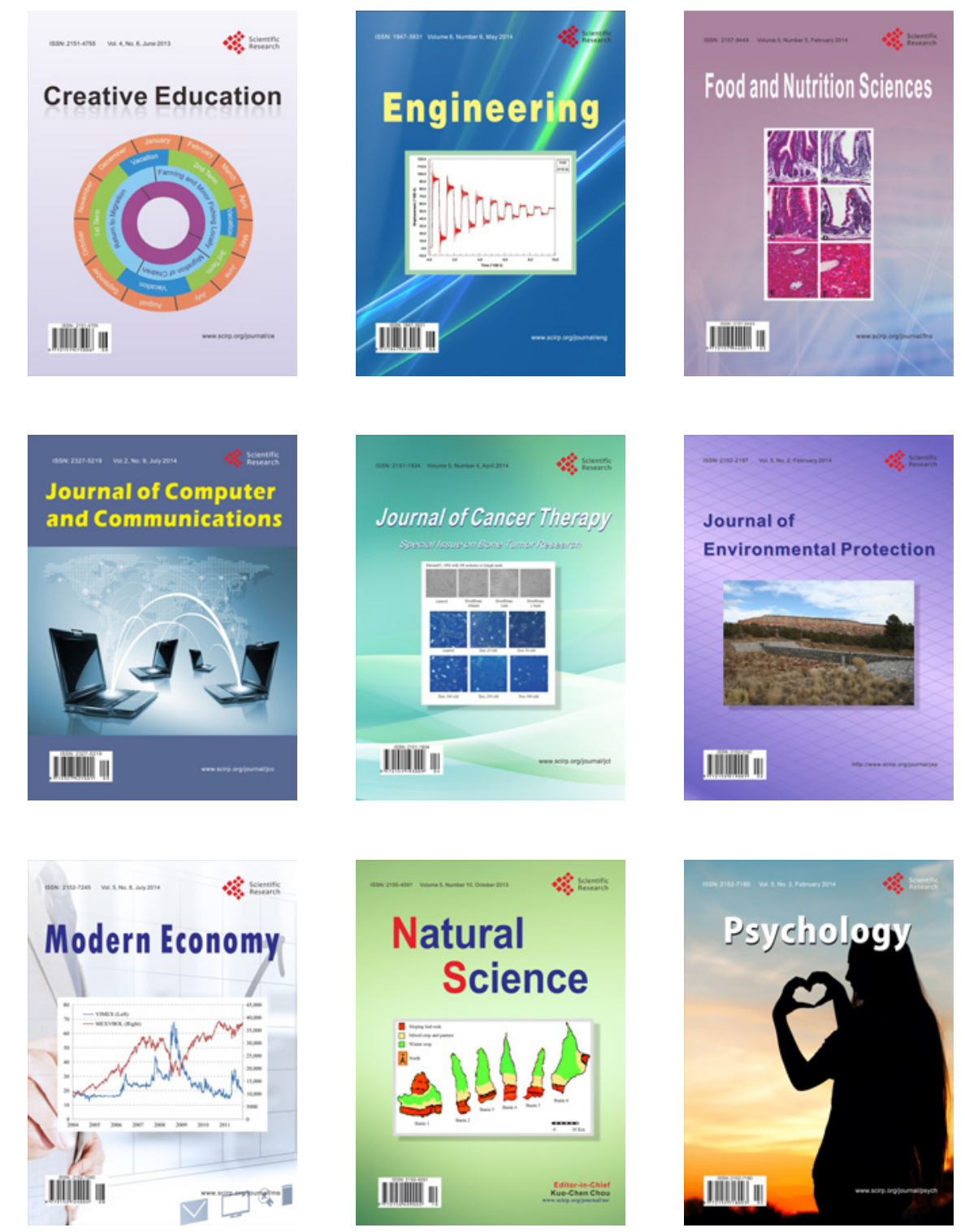